\title{
EQUILÍBRIO LÍQUIDO - LÍQUIDO EM MISTURAS TERNÁRIAS DE LÍQUIDO IÔNICO PRÓTICO BUTIRATO DE 2- HIDROXIETILAMÔNIO + ÁLCOOL (METANOL OU ETANOL) + ACETATO DE PROPILA
}

\begin{abstract}
R. R. PINTO ${ }^{1}$, S. MATTEDI ${ }^{2}$, M. AZNAR ${ }^{1}$
${ }^{1}$ Universidade Estadual de Campinas (UNICAMP), Faculdade de Engenharia Química.

${ }^{2}$ Universidade Federal da Bahia (UFBA), Faculdade de Engenharia Química.

E-mail's para contato: rafarocha2000@gmail.com ou maznar@feq.unicamp.br

RESUMO - Dados de sistemas de equilíbrios líquido-líquido ternários envolvendo líquido iônico prótico Butirato de 2-hidroxietilamônio (2-HEAB) + álcool (metanol ou etanol) + acetato de propila a $25^{\circ} \mathrm{C}$ e pressão atmosférica foram determinados através de duas propriedades, densidade e índice de refração. Os resultados de Equilíbrio Líquido-Líquido mostraram uma pequena região de extração, na parte inferior do diagrama, sendo que o líquido iônico apresentou melhores resultados de extração do metanol do que etanol, com valores percentuais de até $96 \%$. A qualidade da técnica experimental foi analisada através do cálculo da distância entre os pontos de mistura e sua respectiva linha de amarração.
\end{abstract}

\section{INTRODUÇÃO}

Líquidos iônicos pertencem a uma classe de sais orgânicos que, de acordo com uma definição arbitrária, mas amplamente aceita, normalmente são líquidos a temperaturas abaixo de $100^{\circ} \mathrm{C}$. São chamados também de solventes projetáveis, devido à enorme gama de possíveis combinações entre cátions orgânicos e ânions orgânicos ou inorgânicos. Nas últimas décadas esta nova classe de materiais vem atraindo a atenção de pesquisadores que estudam substitutos aos solventes tradicionais, para seu uso devido às suas potenciais aplicações e sua baixa pressão de vapor, segundo Álvarez et al.(2010) e Iglesias et al. (2010), o que minimiza emissões tóxicas ao meio ambiente.

Líquidos iônicos compostos pelo cátion imidazólio são os mais comumente estudados, porém, os líquidos iônicos próticos com base em alquilamônios começaram a se mostrar também atrativos. Líquidos iônicos próticos são formados pela transferência de próton entre uma mistura equimolar de um ácido de Brönsted e de uma base de Brönsted. A principal diferença entre líquidos iônicos próticos e outros líquidos iônicos é a presença de um próton permutável, podendo este produzir ligações de hidrogênio entre o ácido e a base. Autores como Bicak (2004), Cota et al. (2007), Álvarez et al.(2010) e Iglesias et al.(2010) comentam sua síntese simples e sua relevância em importantes processos.

Alguns exemplos do uso dos líquidos iônicos como nova classe de "solventes verdes", segundo Wasserscheid e Keim, (2000) são: operações de separação, onde a purificação de correntes de extrato para regeneração de solvente se dá muitas vezes por destilação devido ao uso de solventes voláteis. Este método só é adequado para a limpeza/regeneração de líquidos iônicos pela evaporação do soluto, uma vez que os líquidos iônicos possuem caráter não volátil à pressão 
atmosférica; e processos onde sistemas aquosos não são possíveis ou mostram sérias desvantagens, pode-se aplicar um líquido iônico como meio polar alternativo a água.

Verificando a importância dos estudos envolvendo esta classe de materiais e devido a escassez de dados de Equilíbrio Líquido - Líquido envolvendo líquidos iônicos próticos, foram estudados os sistemas Butirato de 2-hidroxietilamônio (2-HEAB) + metanol + acetato de propila e Butirato de 2-hidroxietilamônio (2-HEAB) + etanol + acetato de propila a $25^{\circ} \mathrm{C}$ e pressão atmosférica, mapeando suas regiões bifásicas, limitadas por suas tie lines, a fim de verificar o uso deste líquido iônico no processo de separação de álcool em éster.

\section{MATERIAIS E MÉTODOS}

Propriedades, a $25^{\circ} \mathrm{C}$, dos compostos utilizados neste trabalho são apresentadas na Tabela 1. O líquido iônico prótico, Butirato de 2-hidroxietilamônio (2-HEAB) é formado pela base etanolamina e pelo ácido butírico e foi sintetizado em nosso laboratório, de acordo com metodologia segundo Bicak (2004), Cota et al. (2007) e Alvarez et al. (2010). Após 2 meses do líquido iônico sintetizado foi verificado teor de água próximo a 7\%. Para analisar a quantidade de água foi utilizado titulador Karl Fischer volumétrico da Mettler Toledo, modelo DL 31. A fim de diminuir esta quantidade de água o líquido iônico foi purificado a vácuo por 6 horas. Esta operação garantiu teor de água inferior a $1 \%$.

Tabela 1 - Propriedades dos componentes puros a $25^{\circ} \mathrm{C}$

\begin{tabular}{|c|c|c|c|c|c|c|c|}
\hline \multirow[b]{2}{*}{ Componente } & \multirow[b]{2}{*}{$\begin{array}{c}\text { massa molar } \\
(\mathrm{g} / \mathrm{mol})\end{array}$} & \multicolumn{2}{|c|}{ Densidade } & \multicolumn{2}{|c|}{$\begin{array}{l}\text { Índice de } \\
\text { refração }\end{array}$} & \multirow[b]{2}{*}{ Pureza } & \multirow[b]{2}{*}{ Fornecedor } \\
\hline & & Exp & lit & $\exp$ & lit & & \\
\hline Etanolamina & 61.08 & 1.012301 & $1.012700^{\mathrm{a}}$ & 1.4524 & $1.4525^{\mathrm{a}}$ & $99 \%$ & Sigma-Aldrich \\
\hline Ácido Butírico & 88.11 & 0.951644 & $0.952810^{\mathrm{b}}$ & 1.3962 & $1.3961^{\mathrm{b}}$ & $99 \%$ & Sigma-Aldrich \\
\hline 2-HEAB & 149.20 & 1.072588 & $1.072580^{\mathrm{c}}$ & 1.4661 & & $99 \%$ & Sigma-Aldrich \\
\hline Metanol & 32.04 & 0.786654 & $0.786710^{\mathrm{d}}$ & 1.3266 & & $99 \%$ & Sigma-Aldrich \\
\hline Etanol & 46.07 & 0.785076 & $0.785261^{\mathrm{d}}$ & 1.3592 & & $99 \%$ & Sigma-Aldrich \\
\hline Acetato de Propila & 102.13 & 0.882474 & $0.882610^{\mathrm{e}}$ & 1.3818 & & $99 \%$ & Sigma-Aldrich \\
\hline $\begin{array}{l}\text { Murrleta-Guevara e } \\
\text { Bahaduret al., (2013 } \\
\text { Pinto et al. (2013). } \\
\text { Alvarez et al (2011) } \\
\text { Alvarez (2010) }\end{array}$ & driguez ( & & & & & & \\
\hline
\end{tabular}

As propriedades utilizadas para determinar dados de equilíbrio de fases foram densidade, utilizando densímetro Anton Paar DMA- 5000 com precisão de $\pm 5 \times 10^{-6}$, e índice de refração, utilizando refratômetro digital Mettler Toledo RE 40D com precisão de $\pm 1 \times 10^{-4}$. Cada propriedade foi medida a cada ponto de névoa, para determinar as curvas de calibração e também em cada fase de cada tie line, ou linha de amarração, após as misturas atingirem equilíbrio. O método de ponto de névoa foi segundo Iglesias et al. (2007) e De Oliveira e Aznar (2011), utilizando células de equilíbrio encamisadas contendo mistura binária conhecida e gotejando lentamente o terceiro componente, até se obter névoa no sistema. Conhecendo a região de imiscibilidade foram determinados pontos de mistura, contendo os três componentes, sendo que cada ponto de mistura corresponde a uma tie line. Essa mistura conhecida foi pesada diretamente 
dentro da célula de equilíbrio, com o auxílio da balança analítica da Shimadzu, modelo AX 200 e precisão de $0.001 \mathrm{~g}$. Em seguida as células, contendo os três componentes, foram submetidas a forte agitação com auxílio de Vórtex Biomixer, modelo QL 901, a fim de quebrar a tensão superficial entre as fases que foram formadas logo após a pesagem. Quebrada a tensão superficial as misturas foram agitadas nas células por aproximadamente 8 horas, por agitadores magnéticos da Fisatom, modelo 752. Ao final das 8 horas de agitação as células permaneceram em repouso por 12 horas, para atingirem o equilíbrio termodinâmico. Ao atingirem o equilíbrio cada célula apresentou duas fases de aspectos translúcidos e límpidos. Foram coletadas amostras de aproximadamente $3 \mathrm{ml}$ de cada fase para análises das propriedades, $2 \mathrm{ml}$ para análise de densidade e $1 \mathrm{ml}$ para análise de índice de refração. Em todas as etapas dos experimentos, tanto para pontos de névoa como para tie lines, as células estavam conectadas por banho termostático Tecnal TE184 , a fim de garantir o controle de temperatura, em $25^{\circ} \mathrm{C}$.

\section{RESULTADOS E DISCUSSÕES}

Frações mássicas $(w)$, densidades $(\rho)$ e índices de refração $\left(n_{D}\right)$ dos pontos de névoa determinados para fase rica em líquido iônico e rica em éster, são apresentados na Tabela 2.

Tabela 2 - Fração mássica $(w)$, densidade $(\rho)$, índice de refração $\left(\mathrm{n}_{\mathrm{D}}\right)$ para os pontos de névoa para os sistemas 2-HEAB (1) + Metanol (2) + Acetato de Propila (3) e 2-HEAB (1) + Etanol (2) + Acetato de Propila (3) a $25^{\circ} \mathrm{C}$.

\begin{tabular}{|c|c|c|c|c|c|c|c|}
\hline \multicolumn{4}{|c|}{ Metanol } & \multicolumn{4}{|c|}{ Etanol } \\
\hline $\mathrm{w}_{1}$ & $\mathbf{w}_{2}$ & $\mathbf{P}$ & $\mathbf{n}_{\mathrm{D}}$ & $w_{1}$ & $\mathbf{w}_{2}$ & $\rho$ & $\mathbf{n}_{\mathrm{D}}$ \\
\hline 0.8837 & 0.0000 & 1.057106 & 1.4563 & 0.8837 & 0.0000 & 1.057106 & 1.4563 \\
\hline 0.7432 & 0.0956 & 1.014209 & 1.4358 & 0.8119 & 0.0538 & 1.031486 & 1.4463 \\
\hline 0.6712 & 0.1281 & 0.996129 & 1.4278 & 0.6926 & 0.1170 & 0.997269 & 1.4331 \\
\hline 0.6055 & 0.1500 & 0.980782 & 1.4206 & 0.6530 & 0.1326 & 0.987315 & 1.4293 \\
\hline 0.5381 & 0.1679 & 0.965893 & 1.4141 & 0.5703 & 0.1538 & 0.968386 & 1.4216 \\
\hline 0.3600 & 0.1751 & 0.931024 & 1.3994 & 0.5076 & 0.1674 & 0.954949 & 1.4156 \\
\hline 0.2451 & 0.1550 & 0.911205 & 1.3943 & 0.4138 & 0.1725 & 0.936024 & 1.4083 \\
\hline 0.0809 & 0.1030 & 0.886276 & 1.3911 & 0.3138 & 0.1708 & 0.918891 & 1.4006 \\
\hline 0.0204 & 0.0602 & 0.879883 & 1.3830 & 0.2434 & 0.1581 & 0.906831 & 1.3966 \\
\hline \multirow[t]{4}{*}{0.0089} & 0.0000 & 0.882832 & 1.3819 & 0.1877 & 0.1495 & 0.897998 & 1.3924 \\
\hline & & & & 0.0785 & 0.1191 & 0.881993 & 1.3861 \\
\hline & & & & 0.0296 & 0.0789 & 0.877326 & 1.3829 \\
\hline & & & & 0.0089 & 0.0000 & 0.882832 & 1.3819 \\
\hline
\end{tabular}

As curvas de calibração obtidas a partir dos dados da Tabela 2 são apresentados nas Equações 1 e 2 para o sistema: 2-HEAB (1) + Metanol (2) + Acetato de Propila (3); e Equações 3 e 4 para o sistema: 2-HEAB (1) + Etanol (2) + Acetato de Propila (3), ambos a $25^{\circ} \mathrm{C}$ e pressão atmosférica. Os pontos de névoa, que delimitam as regiões homogênea da heterogênea, dos dois sistemas, são apresentados nas Figuras 1 e 2.

$$
\begin{aligned}
& \rho=0.881243+\left(0.187868 . w_{1}\right)-\left(0.092112 . w_{2}\right)+\left(0.110743 . w_{1}\right)^{2}-\left(0.055861 \cdot w_{1} \cdot w_{2}\right) \\
& n_{D}=1.381431-\left(0.009438 . w_{1}\right)+\left(0.071765 . w_{2}\right)+\left(0.326660 . w_{1}\right)^{2}-\left(0.058766 \cdot w_{1} \cdot w_{2}\right) \\
& \rho=0.880997+\left(0.198846 . w_{1}\right)-\left(0.117206 . w_{2}\right)-\left(0.000697 \cdot w_{1}\right)^{2}-\left(0.092918 . w_{1} \cdot w_{2}\right)
\end{aligned}
$$




$$
n_{D}=1.381337+\left(0.061670 \cdot w_{1}\right)-\left(0.000781 \cdot w_{2}\right)+\left(0.161074 \cdot w_{1}\right)^{2}-\left(0.161074 \cdot w_{1} \cdot w_{2}\right)
$$

Os dados de ELL para os sistemas ternários contendo 2-HEAB (1) + Metanol (2) + Acetato de Propila (3) e 2-HEAB (1) + Etanol (2) + Acetato de Propila (3) a $25^{\circ} \mathrm{C}$ e pressão atmosférica, são apresentados na Tabela 3 e 4, respectivamente, juntamente com os coeficientes de distribuição $(K)$ e os coeficientes de seletividade do solvente $(S)$, determinados através das Equações 5 e 6.

$$
\begin{aligned}
& K=\frac{w_{2}^{l i}}{w_{2}^{e s}} \\
& S=\frac{\frac{w_{2}^{l i}}{w_{2}^{e s}}}{\frac{w_{3}^{l i}}{w_{3}^{e s}}}
\end{aligned}
$$

Sendo $\mathrm{w}_{2}$ e $\mathrm{w}_{3}$ frações mássicas dos componentes 2 e 3 , respectivamente e os sobrescritos $l i$

\begin{tabular}{|c|c|c|c|c|c|c|c|c|c|c|c|}
\hline \multicolumn{2}{|c|}{ Misturas } & \multicolumn{4}{|c|}{ Fase rica em Éster } & \multicolumn{4}{|c|}{ Fase rica em 2-HEAB } & \multirow[b]{2}{*}{$\mathbf{K}$} & \multirow[b]{2}{*}{$\mathbf{S}$} \\
\hline$w_{1}$ & $\mathbf{w}_{\mathbf{2}}$ & $\mathrm{w}_{1}$ & $\mathbf{w}_{2}$ & $\mathbf{P}$ & $\mathbf{n}_{\mathrm{D}}$ & $\mathrm{w}_{1}$ & $\mathbf{w}_{2}$ & $\underline{\mathbf{P}}$ & $\mathbf{n}_{\mathbf{D}}$ & & \\
\hline 0.4078 & 0.0249 & 0.0038 & 0.0014 & 0.88208 & 1.3813 & 0.8244 & 0.0395 & 1.03901 & 1.4471 & 29.051 & 212.440 \\
\hline 0.4040 & 0.0356 & 0.0001 & 0.0046 & 0.88168 & 1.3811 & 0.8045 & 0.0610 & 1.03196 & 1.4444 & 13.265 & 98.200 \\
\hline 0.3918 & 0.0546 & 0.0059 & 0.0110 & 0.88114 & 1.3807 & 0.7577 & 0.0864 & 1.01902 & 1.4379 & 7.886 & 49.749 \\
\hline 0.3803 & 0.1047 & 0.0164 & 0.0222 & 0.88019 & 1.3800 & 0.6338 & 0.1501 & 0.98611 & 1.4235 & 6.771 & 30.131 \\
\hline 0.3769 & 0.1330 & 0.0153 & 0.0248 & 0.88063 & 1.3798 & 0.5490 & 0.1669 & 0.96759 & 1.4150 & 6.733 & 22.744 \\
\hline
\end{tabular}
e es representam fase rica em líquido iônico e fase rica em éster, respectivamente.

Tabela 3 - Dados de ELL para o sistema 2-HEAB (1) + Metanol (2) + Acetato de Propila (3) a $25^{\circ} \mathrm{C}$

Tabela 4 - Dados de ELL para o sistema 2-HEAB (1) + Etanol (2) + Acetato de Propila (3) a $25^{\circ} \mathrm{C}$

\begin{tabular}{cccccccccccc}
\hline \multicolumn{2}{c}{ Misturas } & \multicolumn{3}{c}{ Fase rica em Éster } & \multicolumn{7}{c}{ Fase rica em 2-HEAB } \\
$\mathbf{w}_{\mathbf{1}}$ & $\mathbf{w}_{\mathbf{2}}$ & $\mathbf{w}_{\mathbf{1}}$ & $\mathbf{w}_{\mathbf{2}}$ & $\mathbf{P}$ & $\mathbf{n}_{\mathbf{D}}$ & $\mathbf{w}_{\mathbf{1}}$ & $\mathbf{w}_{\mathbf{2}}$ & $\mathbf{P}$ & $\mathbf{n}_{\mathbf{D}}$ & $\mathbf{K}$ & $\mathbf{S}$ \\
\hline 0.3745 & 0.0289 & 0.0011 & 0.0039 & 0.88075 & 1.3814 & 0.7994 & 0.0458 & 1.03117 & 1.4457 & 11.704 & 75.244 \\
0.3598 & 0.0452 & 0.0005 & 0.0091 & 0.87983 & 1.3813 & 0.7819 & 0.0656 & 1.02403 & 1.4433 & 7.170 & 46.554 \\
0.3123 & 0.1052 & 0.0048 & 0.0374 & 0.87756 & 1.3816 & 0.6352 & 0.1470 & 0.98141 & 1.4271 & 3.933 & 17.296 \\
0.3152 & 0.1282 & 0.0133 & 0.0470 & 0.87808 & 1.3821 & 0.5480 & 0.1669 & 0.96189 & 1.4191 & 3.552 & 11.707 \\
\hline
\end{tabular}

Diagramas ternários apresentando curvas binodais e tie lines representam os dados experimentais de ELL, mostrados nas Figuras 1 e 2. Pode-se observar que a região de extração é pequena e se encontra na parte inferior do diagrama, tendo os conjuntos de líquido iônico e metanol ou etanol e acetato de propila e metanol ou etanol totalmente solúveis entre si.Interações moleculares, na fase líquida, normalmente dependem de dois efeitos: variação de forças intermoleculares, ao haver contato entre os componentes, e variações no empacotamento molecular por consequencia de diferentes tamanhos e formatos das moléculas dos componentes. 


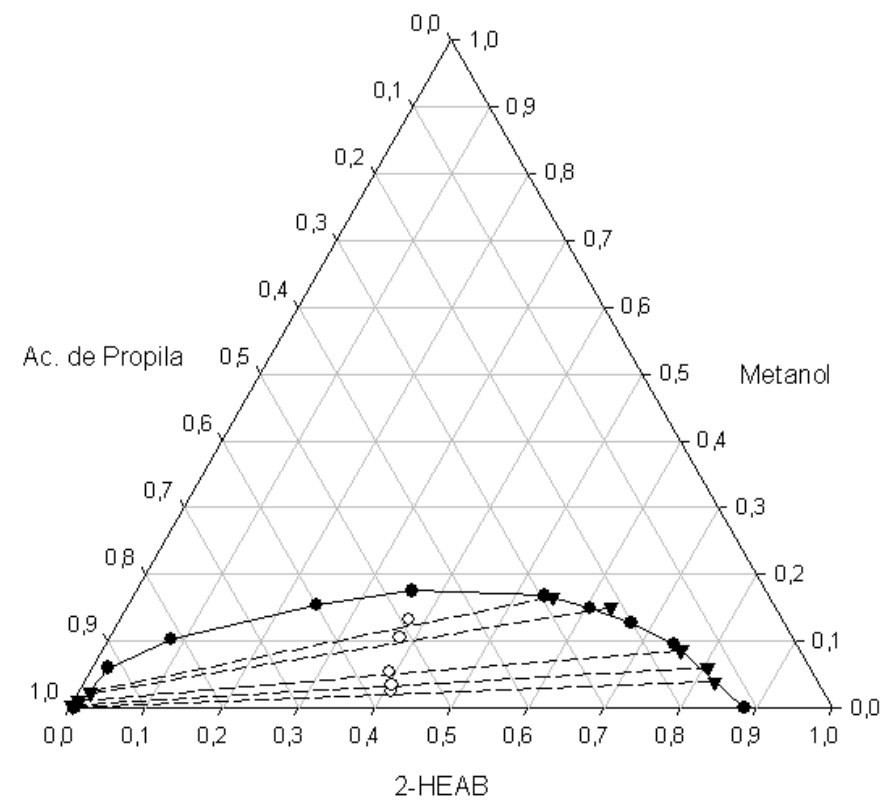

Figura 1 - Diagrama Ternário com Dados Experimentais de Equilíbrio Líquido Líquido para o sistema: 2-HEAB (1) + Metanol (2) + Acetato de Propila (3). (•) pontos de névoa;

( $\circ)$ pontos centrais de mistura; $(\boldsymbol{\nabla})$ frações mássicas dos componentes em equilíbrio.

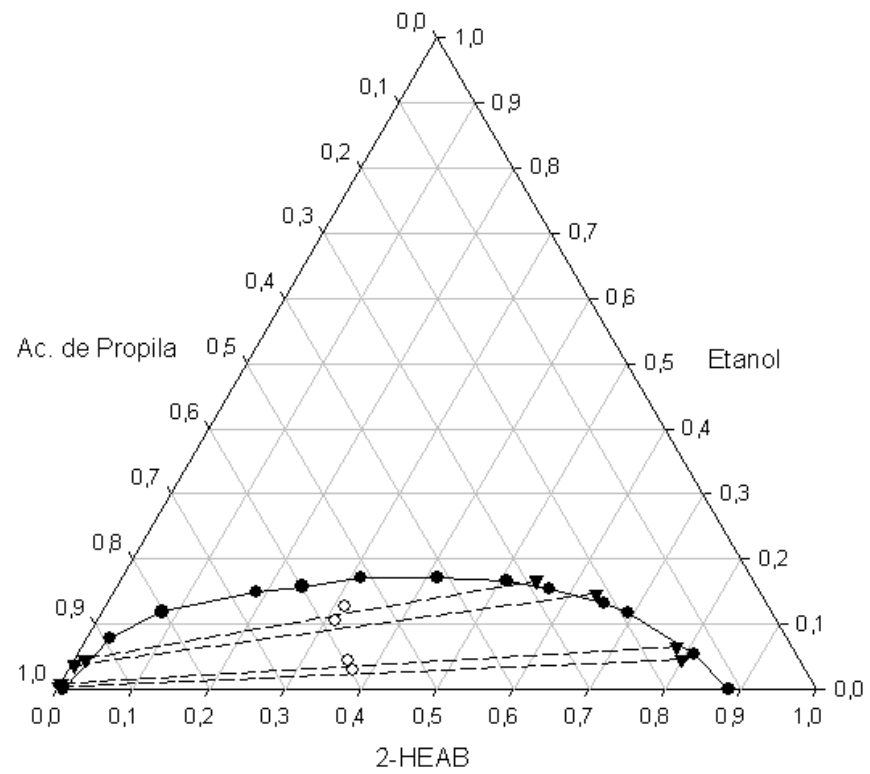

Figura 2 - Diagrama Ternário com dados Experimentais de Equilíbrio Líquido Líquido para o sistema: 2-HEAB (1) + Metanol (2) + Acetato de Propila (3). (•) pontos de névoa;

$(\circ)$ pontos centrais de mistura; $(\boldsymbol{\nabla})$ frações mássicas dos componentes em equilíbrio.

Informações como inclinação das tie lines e seletividades, $K$ e $S$ respectivamente, que são apresentados nas Tabelas 3 e 4, e a distribuição do soluto na fase rica em líquido iônico e na fase rica em éster, podem indicar qual foi o soluto que melhor interagiu com o solvente. As maiores inclinações e coeficientes de seletividade podem ser observadas no sistema contendo metanol. Através da Figura 3 é claro perceber que ambos os alcoóis interagiram mais com o líquido iônico 
do que com o éster, sendo que dentre os dois alcoóis estudados o metanol se distribuiu mais na fase iônica que o etanol

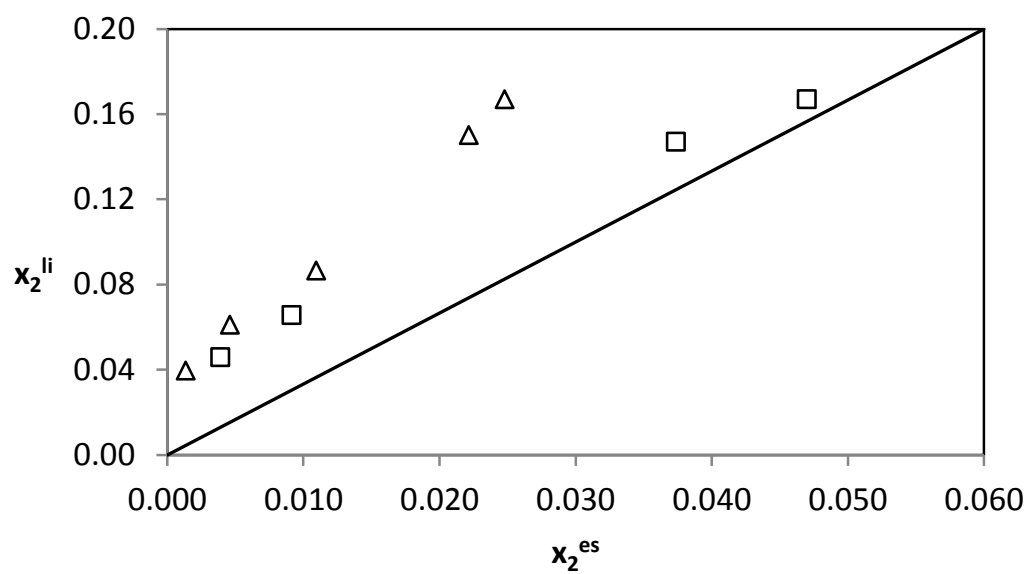

Figura 3. Distribuição dos álcoois entre as fases ricas em líquido iônico (2-HEAB) e éster (Acetato de Propila): metanol $(\Delta)$; etanol $(\square)$.sendo os sitemas: 2-HEAB (1) + Metanol (2) + Acetato de Propila (3) e 2-HEAB (1) + Etanol (2) + Acetato de Propila (3).

A porcentagem de remoção, $E$, do soluto é dada pelas Equações 7 - 9. Os valores de $E$, para cada linha de amarração, são apresentados na Tabela 5 e na Figura 4.

$$
\begin{aligned}
& E=\frac{C_{0}-C_{f}}{C_{0}} \cdot 100 \\
& C_{0}=\left(\frac{w_{2}}{w_{2}+w_{3}}\right)_{\text {misturas }} \\
& C_{f}=\left(\frac{w_{2}}{w_{2}+w_{3}}\right)_{e s}
\end{aligned}
$$

Sendo $\mathrm{C}_{0} \mathrm{e} \mathrm{C}_{\mathrm{f}}$ as frações mássicas do álcool em éster na etapa de mistura e depois de ter obtido equilíbrio, respectivamente. Para os dois sistemas o percentual de extração foi acima de $74 \%$, atingindo os maiores resultados de extração as misturas contendo metanol.

Tabela 5 -Frações mássicas de álcool em acetato de propila antes $\left(\mathrm{C}_{0}\right)$ e após $\left(\mathrm{C}_{\mathrm{f}}\right)$ o equilíbrio e percentuais de extração em cada tie line.

\begin{tabular}{cccccccccc}
\hline \multicolumn{3}{c}{ 2-HEAB + Metanol + Ac. Propila } & \multicolumn{3}{c}{ 2-HEAB + Etanol + Ac. de Propila } \\
\hline Tie line & $\mathbf{w}_{\mathbf{2}}{ }^{\text {mistura }}$ & $\mathbf{C}_{\mathbf{0}}$ & $\mathbf{C}_{\mathbf{f}}$ & $\mathbf{E}$ & Tie line & $\mathbf{w}_{\mathbf{2}}{ }^{\text {mistura }}$ & $\mathbf{C}_{\mathbf{0}}$ & $\mathbf{C}_{\mathbf{f}}$ & $\mathbf{E}$ \\
\hline 1 & 0.0249 & 0.042 & 0.001 & 96.754 & 1 & 0.0289 & 0.046 & 0.004 & 91.513 \\
2 & 0.0356 & 0.060 & 0.005 & 92.294 & 2 & 0.0452 & 0.071 & 0.009 & 87.056 \\
3 & 0.0546 & 0.090 & 0.011 & 87.718 & 3 & 0.1052 & 0.153 & 0.038 & 75.440 \\
4 & 0.1047 & 0.169 & 0.023 & 86.661 & 4 & 0.1282 & 0.187 & 0.048 & 74.563 \\
5 & 0.1330 & 0.213 & 0.025 & 88.209 & & & & & \\
\hline
\end{tabular}


Para verificar a qualidade dos dados foi observado se as tie lines passaram pelos pontos de mistura ao se aplicar uma equação algébrica que mede a distância entre a linha de amarração e o ponto central de mistura. A distância entre o ponto central e sua respectiva tie line é chamado de $u$, e foi obtido através das Equações 10 - 13. Nos trabalhos de De Oliveira e Aznar (2011) estas Equações também foram utilizadas para avaliar os dados experimentais.

$$
\begin{aligned}
& a=x_{2}^{e s}-x_{2}^{l i} \\
& b=x_{1}^{l i}-x_{1}^{e s} \\
& c=x_{1}^{e s} x_{2}^{l i}-x_{1}^{l i} x_{2}^{e s} \\
& u=\frac{\left|a x_{1}^{\text {mistura }}+b x_{2}^{\text {mistura }}+c\right|}{\sqrt{a^{2}+b^{2}}}
\end{aligned}
$$

Os sobrescritos $l i$ e es representam fase rica em líquido iônico e fase rica em éster, respectivamente e o subscrito 2 é referente ao componente 2 do sistema, o álcool. A Tabela 6 mostra os valores de $u$ para cada linha de amarração. A média dos valores de $u$ para o sistema contendo metanol foi menor que a média de $u$ para o sistema com etanol: 0.006 e 0.011, respectivamente. Os valores de $u$ aumentaram na medida em que as linhas de amarração se aproximavam da curva binodal.

Tabela 6 - Distância do ponto central de mistura de sua tie line, $u$,eparâmetros para Equação 10

\begin{tabular}{cccccccccc}
\hline \multicolumn{4}{c}{ 2-HEAB + Metanol + Ac. Propila } & \multicolumn{4}{c}{ 2-HEAB + Etanol + Ac. de Propila } \\
\hline Tie Line & $\boldsymbol{a}$ & $\boldsymbol{b}$ & $\boldsymbol{C}$ & $\boldsymbol{u}$ & Tie Line & $\boldsymbol{a}$ & $\boldsymbol{b}$ & $\boldsymbol{c}$ & $\boldsymbol{u}$ \\
1 & -0.038 & 0.821 & -0.001 & 0.005 & 1 & -0.042 & 0.798 & -0.003 & 0.005 \\
2 & -0.056 & 0.804 & -0.004 & 0.003 & 2 & -0.056 & 0.781 & -0.007 & 0.010 \\
3 & -0.075 & 0.752 & -0.008 & 0.005 & 3 & -0.110 & 0.630 & -0.023 & 0.014 \\
4 & -0.128 & 0.617 & -0.012 & 0.007 & 4 & -0.120 & 0.535 & -0.024 & 0.013 \\
5 & -0.142 & 0.534 & -0.011 & 0.012 & & & & & \\
\hline
\end{tabular}

\section{CONCLUSÕES}

Através de medidas de densidade e índice de refração, diagramas de fases, indicando as regiões de miscibilidade e imiscibilidade, bem como as linhas que indicam as composições em cada fase em equilíbrio, foram determinados para os sitemas 2-HEAB + Metanol + Acetato de Propila e 2-HEAB + Etanol + Acetato de Propila a $25^{\circ} \mathrm{C}$ e pressão ambiente. A consistência dos dados foi verificada através da análise da distância entre a linha de amarração e seu ponto de mistura, onde os valores de $u$ foram baixos. As linhas de amarração mostraram que metanol e etanol são mais solúveis na fase solvente, líquido iônico, do que na fase rafinado, acetato de propila. Os dados experimentais de distribuição do soluto e coeficiente de seletividade mostraram maior interação entre metanol e 2-HEAB, porém o líquido iônico mostrou ser um meio eficaz no processo de extração dos dois álcoois, pois resultados entre 74 e $91 \%$ de extração foram observados para sistema contendo etanol e 88 a $96 \%$ de extração foram encontrados para sitemas contendo metanol. 


\section{AGRADECIMENTOS}

Os autores agradecem a Fundação de Amparo a Pesquisa do Estado de São Paulo - FAPESP (processo 2011-19736/1) e o Conselho Nacional de Desenvolvimento Científico e Tecnológico CNPq.

\section{REFERÊNCIAS}

ALVAREZ, V. H.; MATTEDI, S.; MARTIN-PASTOR, M.; AZNAR, M.; IGLESIAS, M. Synthesis and thermophysical properties of two new protic long-chain ionic liquids with the oleate anion, Fluid Phase Equilibria. 299 (2010) 42-50.

ALVAREZ, V. H.; MATTEDI, S.; MARTIN-PASTOR, M.; AZNAR, M.; IGLESIAS, M. Thermophysical properties of binary mixtures of \{ionic liquid 2-hydroxyethylammonium acetate + (water, methanol, or ethanol) \}.J. Chem. Thermodynamics, 43 (2011) 997-1010.

BAHADUR.I., DEENADAYALU, N., NAIDOO, P., RAMJUGERNATH, D., Density, speed of sound, and refractive measurements for the binary systems (butanoic acid + propanoic acid, or 2methyl-propanoic acid) at $\mathrm{T}=(293.15$ to 313.15) K. J. Chem. Thermodynamics, 57 (2013) $203-211$.

BICAK, N.A new ionic liquid: 2-hydroxy ethylammoniumformate.J. Mol.Liq, 116 (2004) 37-44.

COTA, I.; GONZALES-OLMOS, R.; IGLESIAS, M.; MEDINA, F. New Short Aliphatic Chain Ionic Liquids: Synthesis, Physical Properties, and Catalytic Activity in AldolCondensations.J. Phys. Chem. B 111 (2007) 12468-12477.

DE OLIVEIRA, L. H., AZNAR, M. Liquid - Liquid Equilibria \{1-Ethyl-3-methylimidazolium Diethylphosphate or 1-Ethyl-3-methylimidazolium Ethylsulfate +4 ,6-Dimethyldibenzothiophene + Dodecane Systems at 298.2 K and 313.2 K.J. Chem. Eng. Data, 56 (2011) 2005-2012.

IGLESIAS, M., GONZALES-OLMOS, R., SALVATIERRA, D., RESA, J.M., Analysis of methanol extraction from aqueous solution by $n$-hexane: Equilibruim diagrams as a function of temperature. Journal of Molecular Liquids, 130 (2007) 52 - 58.

IGLESIAS, M.; GONZALES-OLMOS, R.; COTA, I.; MEDINA, F. Brønsted ionic liquids: Study of physico-chemical properties and catalytic activity in aldol condensations. Chemical Engineering Journal. 162 (2010), 802-808.

MURRLETA-GUEVARA, F.; RODRIGUEZ, A. T. Liquid Density as a Function of Temperature of Five Organic Solvents.J. Chem. Eng. Data, 29 (1984), 204-206.

PINTO, R, R,. AZNAR, M., MATTEDI, S., IGLESIAS, M. Propriedades Volumétricas de misturas binárias de três líquidos iônicos próticos + água a $25 \mathrm{E} 50^{\circ} \mathrm{C}$. VII Congresso Brasileiro de Termodinâmica Aplicada, nov. 2013.

WASSERSCHEID, P.; KEIM, W. Ionic liquids - new "solutions" for transition metal catalysis. Angewandte Chemie International Edition. 39, p. 3772-3789. 2000. 